Water on graphene surfaces

This article has been downloaded from IOPscience. Please scroll down to see the full text article.

2010 J. Phys.: Condens. Matter 22284111

(http://iopscience.iop.org/0953-8984/22/28/284111)

View the table of contents for this issue, or go to the journal homepage for more

Download details:

IP Address: 147.83.54.94

The article was downloaded on 22/06/2010 at 15:00

Please note that terms and conditions apply. 


\title{
Water on graphene surfaces
}

\author{
M C Gordillo ${ }^{1,3}$ and J Martí ${ }^{2}$ \\ ${ }^{1}$ Departamento de Sistemas Físicos, Químicos y Naturales, Facultad de Ciencias \\ Experimentales, Universidad Pablo de Olavide, Carretera de Utrera, km 1, E-41013 Sevilla, \\ Spain \\ 2 Departament de Física i Enginyeria Nuclear, Universitat Politècnica de Catalunya, \\ B4-B5 Campus Nord, E-08034 Barcelona, Catalonia, Spain \\ E-mail: cgorbar@upo.es and jordi.marti@upc.edu
}

Received 20 November 2009, in final form 12 February 2010

Published 21 June 2010

Online at stacks.iop.org/JPhysCM/22/284111

\begin{abstract}
In this paper, we summarize the main results obtained in our group about the behavior of water confined inside or close to different graphene surfaces by means of molecular dynamics simulations. These include the inside and outside of carbon nanotubes, and the confinement inside a slit pore or a single graphene sheet. We paid special attention to some thermodynamical (binding energies), structural (hydrogen-bond distributions) and dynamic (infrared spectra) properties, and their comparison to their bulk counterparts.
\end{abstract}

\section{Introduction}

Confined water is important in a wide range of physical situations, such as in electrochemical systems [1, 2], solar energy conversion [3], meteorology [4], corrosion processes [5, 6], heterogeneous catalysis of metals [7] and in biological systems [8], just to name a few. In particular, it has been observed that the microscopic structure, the dielectric properties and the dynamics of water suffer important changes when water is close to solid or liquid interfaces [9]. We can also have important changes in the thermodynamical stability limits of constrained or adsorbed water [10, 11].

From the theoretical and computational side, many works [12-16] have employed averaged effective potentials to model water-surface forces in a rather coarse-grained way, although more specific systems have been widely studied as well: water close to or inside silica pores (Vycor glass) [17], platinum [18], magnetite [19], zirconia [20], mica [21] or pure carbon composites (graphite structure) in several geometries, from planar [22-26] to cylindrical [27-29]. A detailed review of recent developments in the field from a computational perspective was reported by Zangi [30]. From the experimental side, many authors have contributed to the study of hydrophobic-water interfaces by means of a variety of techniques, among them scanning tunneling microscopy [31], atomic force spectroscopy [32], neutron diffraction experiments [33-36], calorimetry [37] or ultrafast optical Kerr effect spectroscopy [38].

\footnotetext{
3 Author to whom any correspondence should be addressed.
}

In this work, we will summarize our work on confined water in different geometries, ranging from carbon nanotubes (a rolled-up graphene layer) to slit flat graphene pores and ending with the study of water on top of a single graphene sheet [39], i.e. we studied the confining effect in water for different geometries for the same type of surface. In all cases, we considered a wide range of thermodynamical conditions, from ambient-like to supercritical. Special attention was paid to the thermodynamical stability of the systems studied by means of the corresponding adsorption free energies obtained by the proper least-squares fitting procedure [28]. This allowed us to compute structural properties for the stable phases, such as atomic density profiles, molecular orientations and hydrogen-bond distributions that were shown to be quite different than their bulk counterparts. The same could be said of the dynamical properties of confined water, with important changes in translational and rotational diffusion coefficients $[15,16,40]$ or in the infrared spectrum $[35,41-43]$.

\section{Models and simulation details}

In all the cases presented below, we considered water molecules to be well represented by a flexible SPC (simple point charge potential) [44] modified to reproduce the location of the maxima of the main infrared (IR) spectral bands. This is an empirical model, originally defined by Toukan and Rahman [45], whose reparameterization was successfully tested for water in a wide variety of thermodynamical conditions [46, 47]. Water-carbon interactions have been 


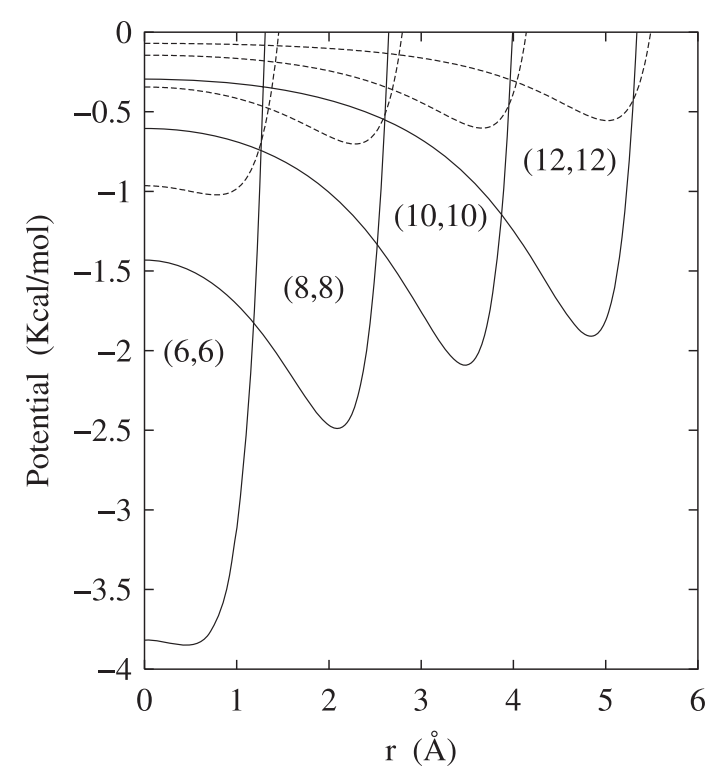

Figure 1. Oxygen-tube (full lines) and hydrogen-tube (dashed lines) interaction for different carbon nanotubes.

modeled by means of a Lennard-Jones model [48] where individual carbon-oxygen and carbon-hydrogen interactions were obtained through application of the Lorentz-Berthelot rules. Unless we say something else, all the individual carbonwater interactions were considered. Long-ranged interactions have been considered by means of the Ewald sum rule in all systems.

Our molecular dynamics (MD) simulations used a leapfrog Verlet integration algorithm coupled to a thermal bath [49] to keep the temperature fluctuating within a reasonable range (around 10\% of average values). The integration time step was $0.5 \mathrm{fs}$, with equilibration periods of 100 ps. Averages of dynamical quantities were computed from runs of at least $250 \mathrm{ps}$.

\section{Results}

We will start by considering water in cylindrical confinement. In practice, this is easily done by introducing it inside carbon nanotubes of different radii. Carbon nanotubes are cylinders that can considered to be the result of 'cutting' a strip from a graphene sheet and rolling it up in such a way as to leave no dangling bonds [50]. Since the strip can be cut with any orientation, this could produce different kinds of nanotubes with different radii, of which we will concern ourselves only with those of the $(n, n)$ type in the standard nomenclature [51], in particular with the ones in table 1, which also includes the number of water molecules $\left(N_{\mathrm{w}}\right)$ considered in a simulation cell of $74.5 \AA$ to obtain a reference density inside the tube of $1 \mathrm{~g} \mathrm{~cm}^{-3}$. The potential used was of Lennard-Jones type, but we did not considered all the $\mathrm{C}$-water interactions, but the averaged interaction of Stan and Cole [52], in which any $\mathrm{O}$ and $\mathrm{H}$ atoms interact with the nanotube as a whole. These potentials are depicted in figure 1 and are very close to the result of considering all the $\mathrm{C}$ atom interactions. The distance

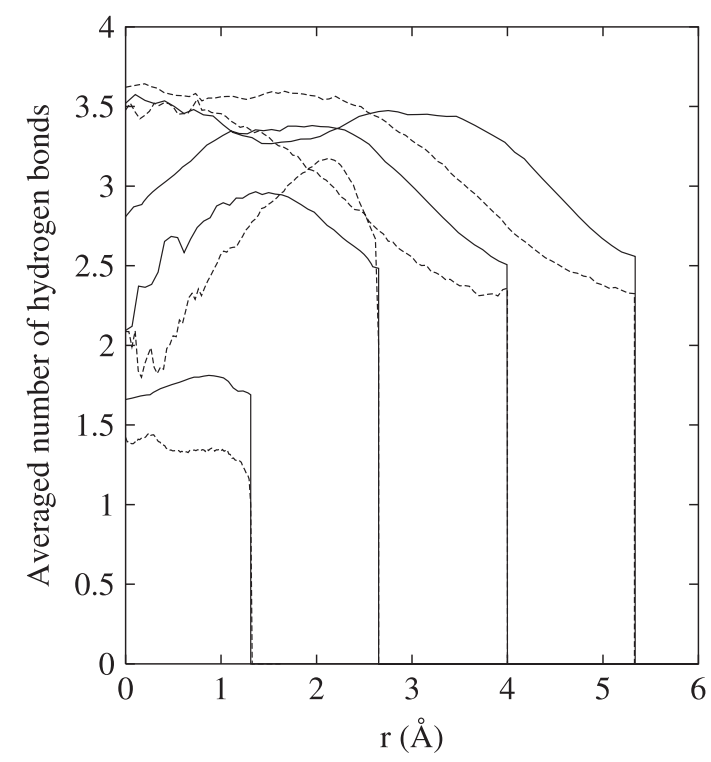

Figure 2. Average number of H-bonds as a function of distance from the center of the tube. Carbon tubes are represented by continuous lines and rigid cylinders by dashed lines. The represented tubes are $(6,6),(8,8),(10,10)$ and $(12,12)$.

Table 1. Summary of carbon nanotubes used as confining devices.

\begin{tabular}{lllr}
\hline Tube radius $(\AA)$ & Internal radius $(\AA)$ & Tube class & $\left(N_{\mathrm{w}}\right)$ \\
\hline 4.10 & 1.31 & $(6,6)$ & 14 \\
5.45 & 2.65 & $(8,8)$ & 56 \\
6.80 & 4.00 & $(10,10)$ & 126 \\
8.15 & 5.33 & $(12,12)$ & 224 \\
\hline
\end{tabular}

from the center of the tube at which the oxygen-tube potential is zero is given in table 1 as the internal radius, and indicates the effective space available to water. The density of $1 \mathrm{~g} \mathrm{~cm}^{-3}$ was obtained by taking into account only this available space.

The main structural parameter we are interested in is the structure of the hydrogen-bond network, which can be deduced with the help of figure 2 in which we display the average number of $\mathrm{H}$-bonds of a molecule located at a distance $r$ of the center of each cylinder at $298 \mathrm{~K}$. At this temperature and at a density of $1 \mathrm{~g} \mathrm{~cm}^{-3}$ water is a liquid with a welldefined layered structure [42, 48, 53, 54], which would start to freeze at different temperatures depending on the diameter of the carbon nanotube, but in any case below $300 \mathrm{~K}$ [55]. The number of hydrogen bonds that any particular molecule forms is calculated by means of a geometrical criterion [48]. To separate the effects of pure confinement to those of the external potential, we present the cases of carbon nanotubes (full lines) and purely rigid cylinders of the same effective radius (internal radius in table 1 , dashed lines). In both cases, we observe a decreasing, some times considerable, of the average number of hydrogen bonds per molecule with respect to the case of bulk water under the same conditions (3.7). This decrease is especially important in the case of the tube with a inner diameter of $2.31 \AA((6,6)$ in the standard nomenclature), in which it is impossible to have two molecules in the same $x y$ plane perpendicular to the main 


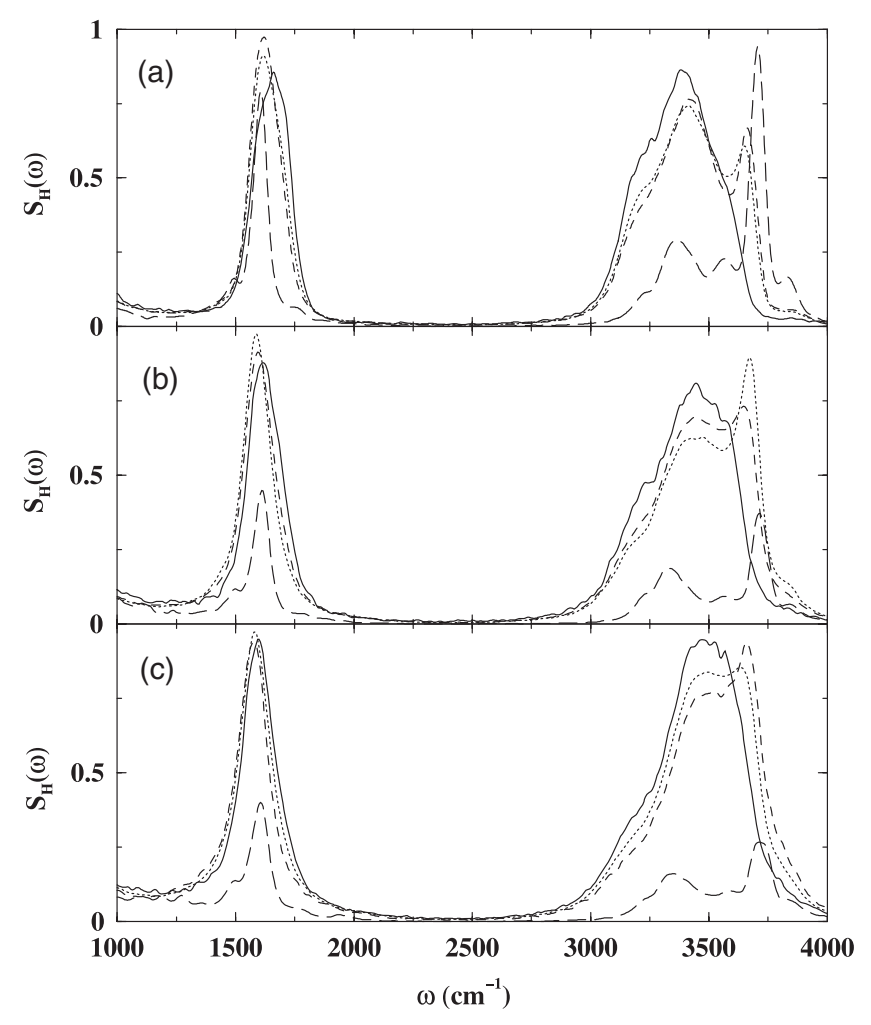

Figure 3. $S_{\mathrm{H}}(\omega)$ s for water confined inside carbon nanotubes at different temperatures: (a) $298 \mathrm{~K}$, (b) $400 \mathrm{~K}$ and (c) $500 \mathrm{~K}$. Full lines, bulk water; long dashed lines, $(6,6)$ tube; dashed lines, $(8,8)$ tube and dotted lines $(10,10)$ tube.

tube axis. The fact that the number of H-bonds is greater in the carbon nanotube case when we are closer to the cylinder walls is due to the greater accumulation of molecules at the external potential minima displayed in figure 1 . The anomalous case of the $(8,8)$ tube, in which the opposite happens, is due to the competition between the location of the external potential minimum and the minimum of the intermolecular $\mathrm{O}_{-}$ O potential in an environment where the system tries to squeeze more water molecules into the $x y$ plane to maximize the overall number of H-bonds.

Arguably, one of the most interesting dynamical features of water is its infrared spectrum. It is well known that one can use the hydrogen velocity autocorrelation functions to compute the spectral densities $S_{\mathrm{H}}(\omega)$, which are directly related to these spectra [41, 42]. In particular, the maxima of $S_{\mathrm{H}}(\omega)$ should coincide with the position of a real spectroscopic band.

The wavelength range between 1000 and $4000 \mathrm{~cm}^{-1}$ is displayed in figure 3 . In this interval, we have the frequencies corresponding to the bending $\left(\sim 1600 \mathrm{~cm}^{-1}\right)$ and stretching $\left(\sim 3400 \mathrm{~cm}^{-1}\right)$ bands for three different temperatures: from top to bottom we have (a) $298 \mathrm{~K}$, (b) $400 \mathrm{~K}$ and (c) $500 \mathrm{~K}$. In all cases, full lines correspond to bulk water, long dashed lines to a $(6,6)$ tube, dashed lines to an $(8,8)$ tube and dotted lines to a $(10,10)$ one. In all cases, we observed that the bending band is slightly backshifted with respect to the bulk value at all temperatures, but the changes are minimal when we vary the tube. The most significant feature of the spectra is the splitting of the stretching band, with a maximum around the bulk values,
Table 2. Average number of hydrogen bonds per water molecule.

\begin{tabular}{lllllll}
\hline & & \multicolumn{5}{c}{$\rho\left(\mathrm{g} \mathrm{cm}^{-3}\right)$} \\
\cline { 3 - 7 } Tube & $T(\mathrm{~K})$ & 0.83 & 0.72 & 0.66 & 0.49 & 0.26 \\
\hline$(12,12)$ & 573 & 1.43 & 1.30 & & & \\
& 673 & 1.10 & & 0.93 & 0.72 & \\
& 773 & 1.02 & & & & 0.27 \\
$(10,10)$ & 573 & 1.23 & 1.16 & & & \\
& 673 & 0.95 & & 0.89 & 0.61 & \\
& 773 & 0.88 & & & & 0.23 \\
$(8,8)$ & 573 & 1.07 & 0.88 & & & \\
& 673 & 0.69 & & 0.65 & 0.43 & \\
& 773 & 0.64 & & & & 0.17 \\
Bulk & Average & 2.5 & 2.4 & 2.1 & 1.6 & 0.8 \\
\hline
\end{tabular}

Table 3. Frequency maxima (in $\mathrm{cm}^{-1}$ ) of the stretching band in SW water confined in carbon nanotubes.

\begin{tabular}{lllllll}
\hline & & \multicolumn{6}{c}{$\rho\left(\mathrm{g} \mathrm{cm}^{-3}\right)$} \\
\cline { 3 - 7 } Tube & $T(\mathrm{~K})$ & 0.83 & 0.72 & 0.66 & 0.49 & 0.26 \\
\hline$(12,12)$ & 573 & - & 3625 & & & \\
& 673 & - & & 3640 & - & 3770 \\
& 773 & - & & & & \\
$(10,10)$ & 573 & 3635 & 3635 & & & \\
& 673 & 3635 & & - & 3700 & \\
& 773 & - & & & & 3810 \\
$(8,8)$ & 573 & 3680 & 3685 & & & \\
& 673 & 3670 & & 3695 & 3720 & \\
& 773 & 3675 & & & & 3855 \\
Bulk & 573 & 3500 & 3520 & & & \\
& 673 & 3510 & & 3515 & 3545 & \\
& 773 & 3495 & & & & 3555 \\
\hline
\end{tabular}

and another one at higher wavenumbers. We found that the splitting was proportional to the inverse of the radius of the nanotube, at least at room temperature [41]. The splitting itself was due to the appearance of molecules that are not hydrogenbonded, producing the high wavelength band [22], and it can also be seen for heavy water under the same conditions [43]. This splitting can also be seen in the simulation results of Mann and Halls [56] for water inside a $(6,6)$ tube. The shifting of the stretching band to higher wavenumbers due to confinement was confirmed experimentally for ice inside nanotubes [35].

To complete the study of water in nanotubes, we will also show some results [57-59] for the same properties studied above but for supercritical water (SW) by means of tables 2 and 3. What we can see is that the number of hydrogen bonds decreases considerably with respect to the room temperature conditions due to the combined effect of the reduction in the density and the increase in the temperature. In fact, the average number of bonds per molecule decreases below one in many cases, indicating that the molecules do not even form linear chains, but smaller groups. This is also reflected in the disappearance of the splitting in the stretching band, probably due to the fact that the majority of molecules are non-bonded. This is supported by the fact that the maxima in table 3 are located at similar values than the high wavenumber band at lower temperatures. 


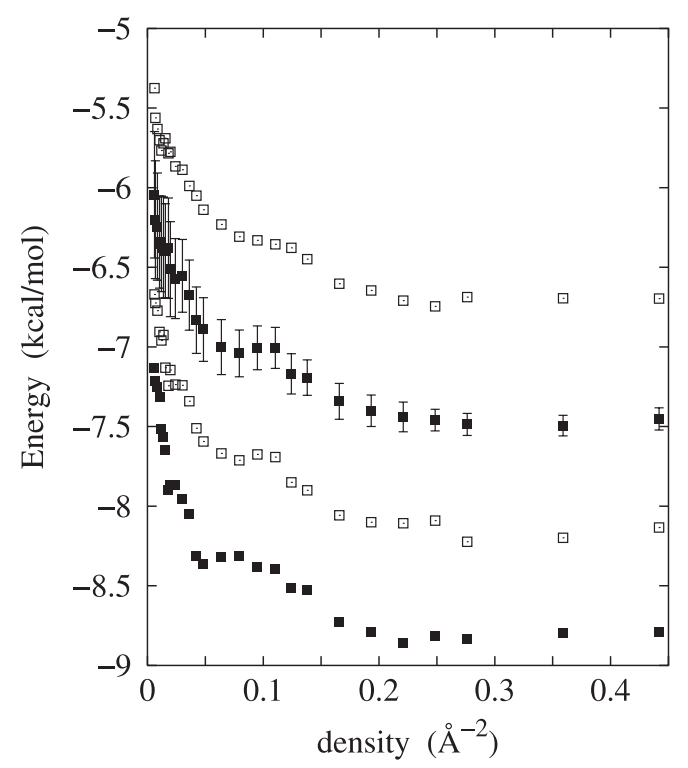

Figure 4. Adsorption energy as a function of density for (from top to bottom) $323,298,273$ and $250 \mathrm{~K}$ for water on top of a bundle of carbon nanotubes.

All the above results are possible only if the nanotubes are open by mechanical or chemical ways, the reason being that these structures are usually synthesized as closed ones [51]. Moreover, the carbon nanotubes usually associate to form structures called bundles, of which we are certain that they possess an outer surface fully accessible to adsorbates, in particular, water. In fact, one can see that water binds to this substrate by studying the adsorption energy of water on top of it, as displayed in figure 4. From top to bottom we have the results for 323, 298, 273 and $250 \mathrm{~K}$. Since in all cases we have negative values, we conclude that water adsorbs on top of these structures $[28,60]$.

However, from this information alone we cannot know if water has any thermodynamical stable phases on top of a bundle of carbon nanotubes. To obtain this information, we need to know the free energy of the system, and not merely its energy, as displayed in figure 4 . This, however, can be done by the fitting procedure outlined in [28]; we assumed a reasonable form for the free energy, $F$, such as for instance:

$$
F(\rho, T)=\sum_{i=0}^{3} \sum_{j=1}^{3} b_{i j} \rho^{i} T^{1-j}+\text { ideal polyatomic gas terms. }
$$

( $\rho$, water density in $\AA^{-2}$ ) and then derived the corresponding form for the total energy of the system, $E$, a property that can be obtained directly from molecular dynamics simulations and the basic relations of thermodynamics. The fitting of the results given in figure 4 to the energy derived from equation (1) allowed us, going backwards, to obtain the free energy for water on top of a bundle of carbon nanotubes.

The results of that fitting procedure are given in figure 5 . There, we represent $F$ as a function of the inverse of the density for the data at four temperatures displayed in figure 4 . The dotted lines are the corresponding double tangent lines of a

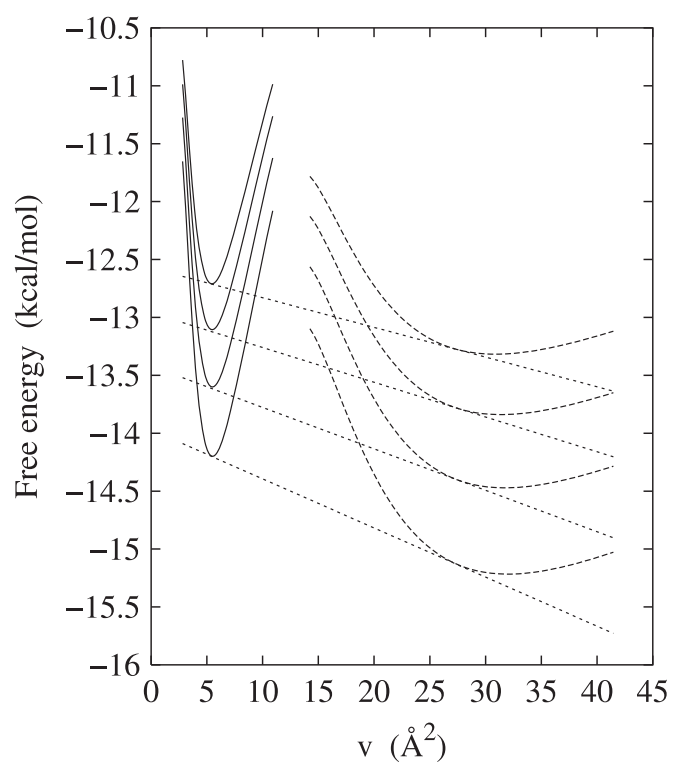

Figure 5. Free energy as a function of the inverse of the water density. From top to bottom 250, 273, 298 and $323 \mathrm{~K}$.

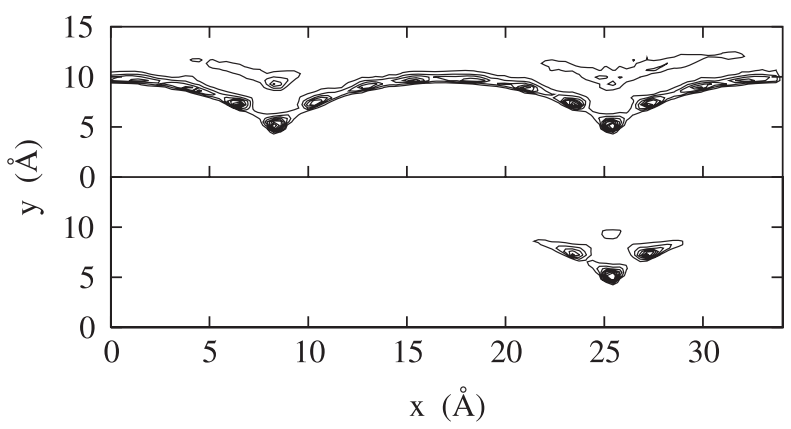

Figure 6. High density (upper panel) and low density (lower panel) water phases on top of a bundle of carbon nanotubes.

Maxwell construction, that allows us to define two phases, one at densities above $\rho=0.18 \AA^{-2}$ and another around $\rho=0.036 \AA^{-2}$. A density contour plot for both phases, shown in figure 6 , indicates that the upper density one corresponds to a water monolayer and the other to a phase in which the water molecules are concentrated in the space between the cylinders, in what is called a groove.

An identical procedure was used to study what happens to water inside slit pores limited by single graphene sheets separated by a given distance $d$. Both for relatively narrow [26, 61] $(d \leqslant 15 \AA)$ and wider [62-64] $(d=31 \AA)$ pores. For instance, in figure 7 we present the raw data we used to obtain the corresponding free energies for slit pores with $d=7,9,12$ and $15 \AA$. In the temperature range chosen, the water was a liquid, as confirmed independently by the phase diagrams of confined water between hydrophobic plates of $[65,66]$. These data of figure 7 allow us to obtain phase diagrams like the one presented in figure 8 , in which we can see that, for $d=12 \AA$, there is no water stable up to relatively high densities. We can see also that the critical point for water in this set-up has to be located at higher temperatures than the 

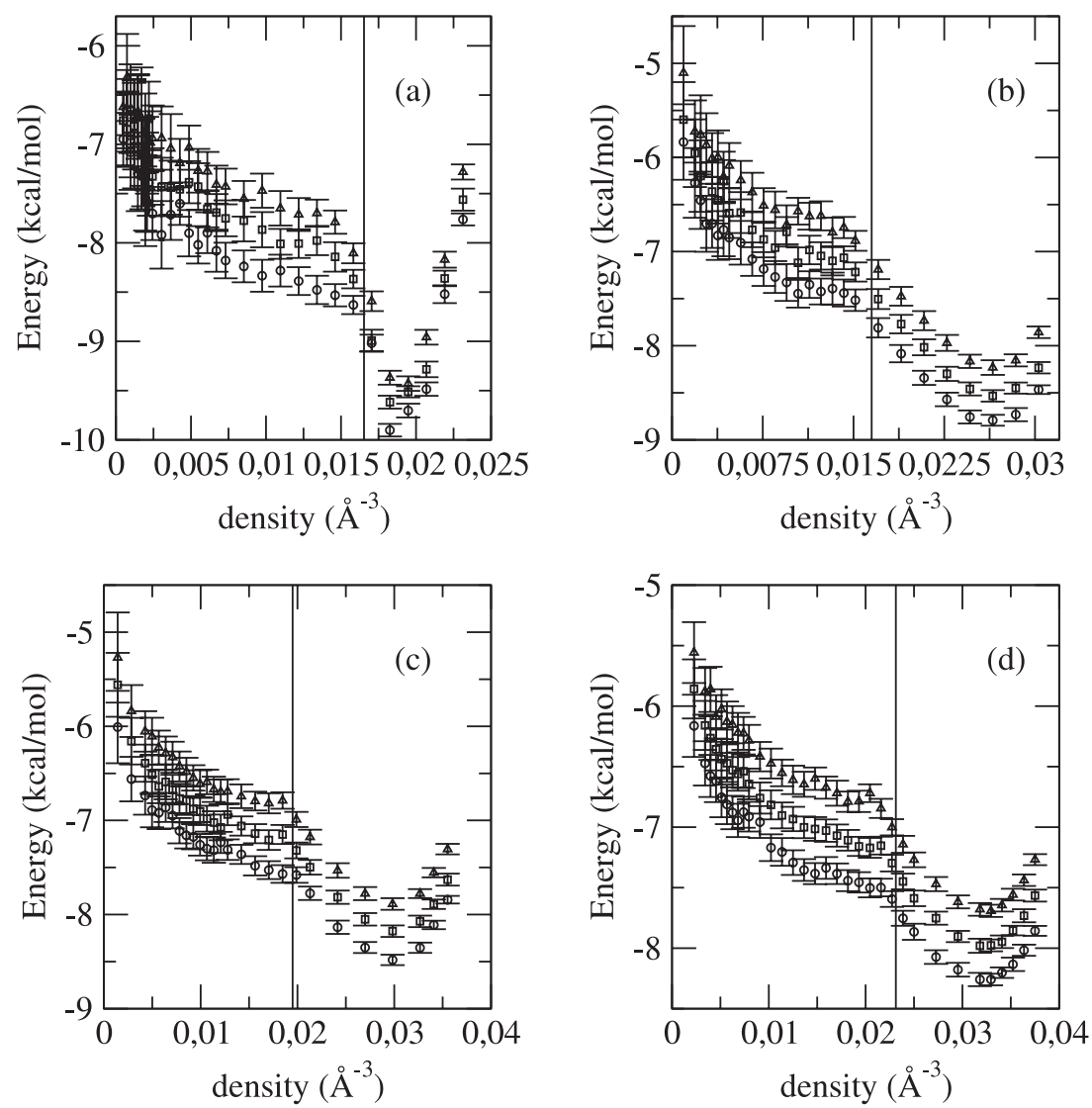

Figure 7. Energy per water molecule inside different slit pores: (a) $d=7 \AA$; (b) $d=9 \AA$; (c) $d=12 \AA$; (d) $d=15 \AA$. In all cases, circles correspond to simulations at $298 \mathrm{~K}$, squares at $310 \mathrm{~K}$ and triangles at $323 \mathrm{~K}$.

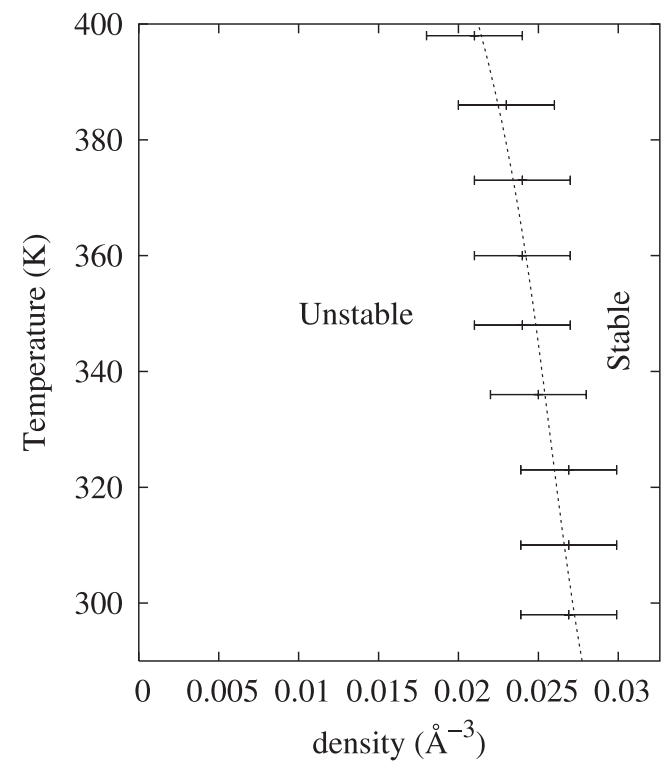

Figure 8. Phase diagram for water inside a $d=12 \AA$ width slit pore.

ones displayed, as happens in wider slit-like pores [67]. As in other cases of confinement, the number of hydrogen bonds decreases, both from the confinement itself and the temperature increase, something not true for the infrared spectra, which behaves similarly than in bulk water [61].
On the other hand, a study on the stability of water inside pores with $d=31 \AA$ allows us to establish the existence of a low density phase, characterized by a double water layer close to the walls, and an bulk-like one, in which water fills all the available space in the slit pore. The density profiles at $298 \mathrm{~K}$ of both phases are displayed in figures 9 and 10 for densities 0.22 and $1.05 \mathrm{~g} \mathrm{~cm}^{-3}$, respectively. In the second case, as figure 10 indicates, we can distinguish three different water zones: bulk, intermediate and interfacial, all of them characterized by different behaviors in the water orientation, something that can be seen with the help of figure 11. There, we can see the angle formed by the water dipole and any of the $\mathrm{OH}$ vectors with a unit vector perpendicular to the graphene surface [68]. From these results, we can deduce that there are two preferred orientations for water close to the walls: one in which one of the $\mathrm{OH}$ points outward (typical of a waterhydrophobic surface interaction) and another one in which one of the bonds is basically parallel to the surface. This is so for water in any of the phases in the slit pore [26, 62, 69] and also for water on top of a single graphene sheet [70], and is corroborated by the dipole probability profile.

A quantity that can be computed by water inside a slit pore is its dielectric constant. This can be obtained by means of the following expression:

$$
\epsilon=1+\frac{4 \pi\left\langle M^{2}\right\rangle}{3 V k_{\mathrm{B}} T}
$$




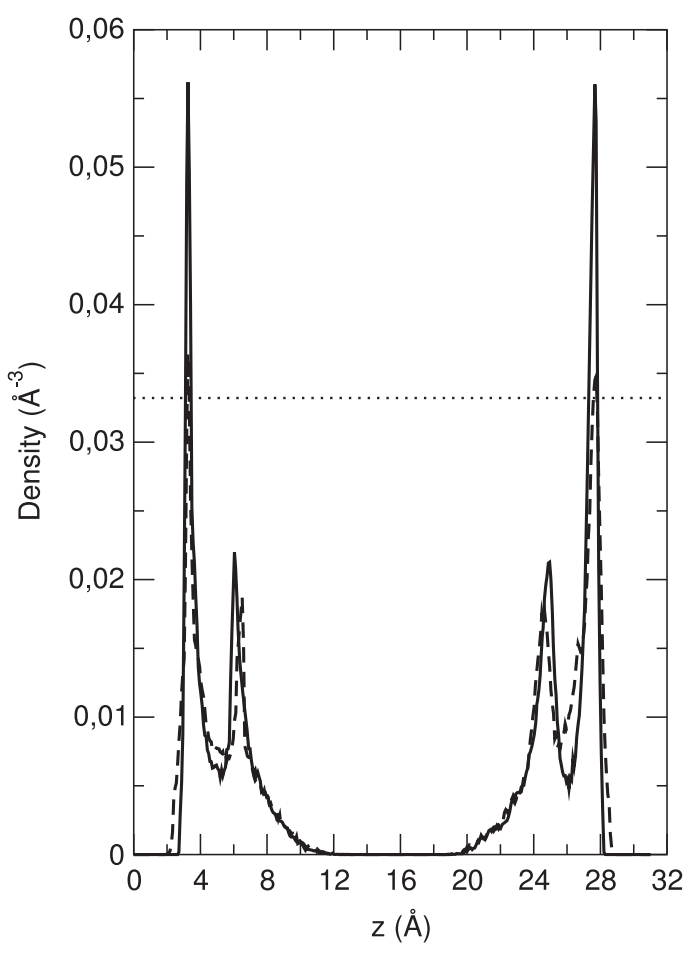

Figure 9. Oxygen (full line) and hydrogen (dashed line) density profiles for a stable low density phase inside a slit pore with $d=31 \AA$.

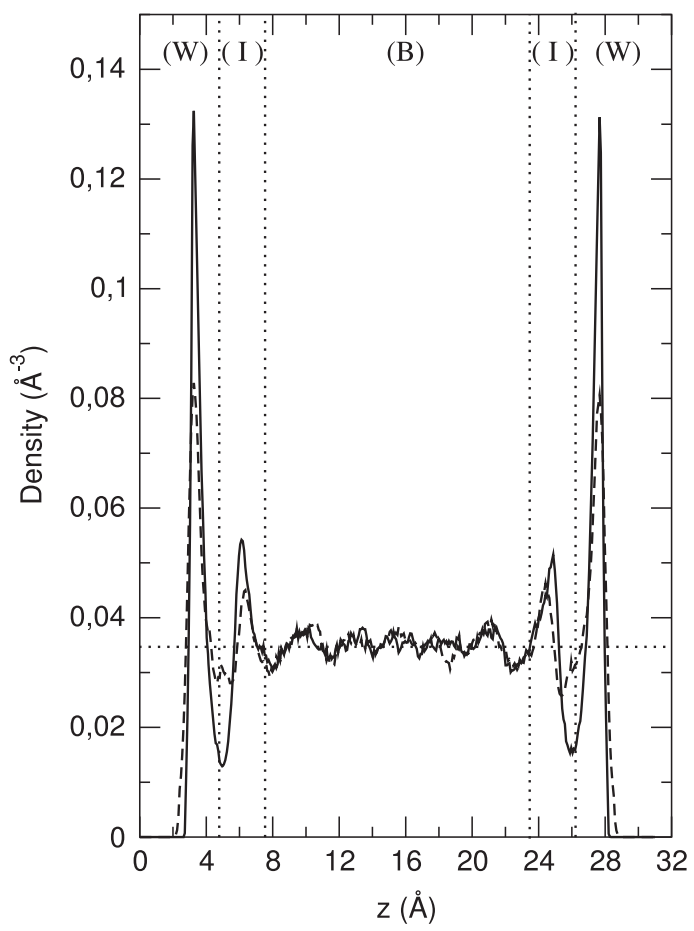

Figure 10. Same as in the previous figure for a high density phase. The horizontal line indicates a bulk density phase for that temperature $(298 \mathrm{~K})$. B means bulk-like, I intermediate and W interfacial water.

where $V$ is the volume of the simulation cell, $k_{\mathrm{B}}$ is the Boltzmann constant and $\left\langle M^{2}\right\rangle$ is the average total dipole moment of the system. The results for three temperatures and

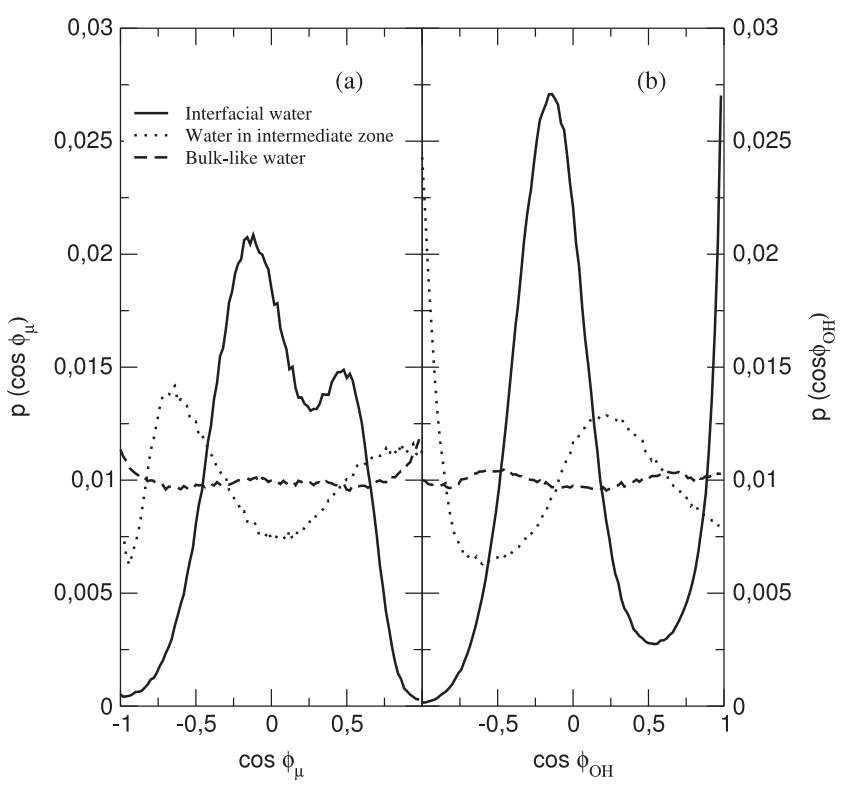

Figure 11. (a) Water dipole and (b) $\mathrm{OH}$ orientations with respect to the closest graphene wall.

Table 4. Static dielectric constant of confined water in different regions.

\begin{tabular}{lllrc}
\hline$T(\mathrm{~K})$ & Density $\left(\mathrm{g} \mathrm{cm}^{-3}\right)$ & Region & \multicolumn{2}{c}{ \% occupation $\epsilon$} \\
\hline 298 & 1.000 & Adsorbed & 23 & $172 \pm 95$ \\
& & Intermediate & 17 & $30 \pm 8$ \\
& & Bulk & 60 & $35 \pm 5$ \\
473 & \multirow{4}{*}{0.863} & Total & 100 & $85 \pm 25$ \\
& & Adsorbed & 20 & $34 \pm 5$ \\
& & Intermediate & 19 & $19 \pm 5$ \\
473 & \multirow{4}{*}{0.526} & Bulk & 61 & $30 \pm 3$ \\
& & Total & 100 & $27 \pm 3$ \\
& & Adsorbed & 20 & $8.3 \pm 0.4$ \\
& & Bulk & 80 & $5.8 \pm 0.2$ \\
& & Total & 100 & $5.8 \pm 0.2$ \\
\hline
\end{tabular}

densities are given in table 4, the last one corresponding to a supercritical state. In this last case, there were observed no differences between interfacial and bulk water [64, 71]. At room temperature, the values of $\epsilon$ are larger in the adsorbed regions, and to decrease in the intermediate and bulk zones, in good agreement with previous results [72]. However, at higher temperatures, the difference decreases considerably due to the strong molecular disorder that induced a loss of orientational order in the water dipoles.

The average number of hydrogen bonds close to a wall decreases considerably close to the wall $[26,62,70]$, as can be seen in figure 12 in the case of a single graphene sheet. There, we display the density profile for a water density of $0.85 \AA^{-2}$ (full line) together with the average number of hydrogen bonds at a given distance. This is fairly representative of the situations for which the absorbed water is thermodynamically stable $\left(\rho \geqslant 0.29 \AA^{-2}\right)$. We can see that the number of hydrogen bonds is around three close to the wall or to the void, and in the intermediate region increases to $\sim 3.5$, a number similar to the one in bulk. The number of bonds close to the wall stems 


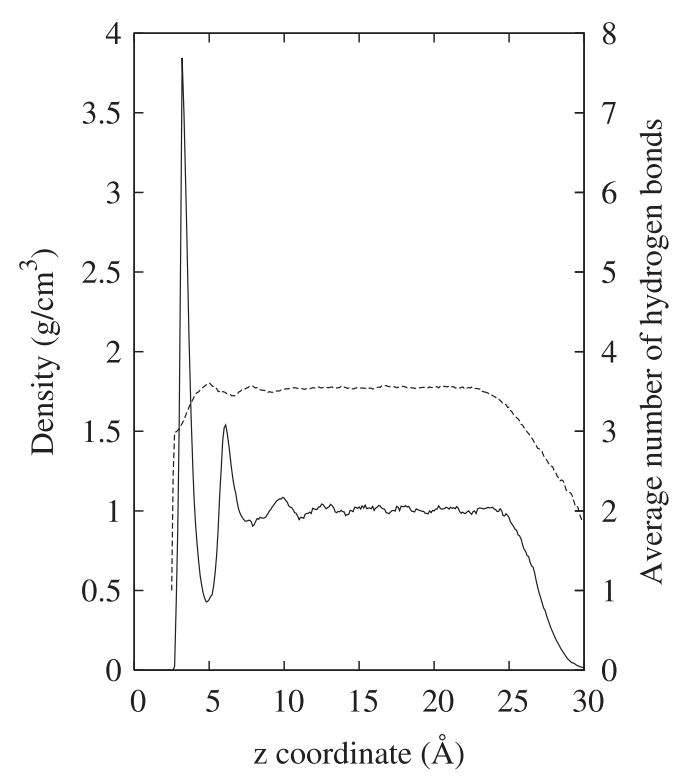

Figure 12. Average hydrogen-bond number for a single graphene sheet (dashed line) and water density profile at $298 \mathrm{~K}$ (full line).

from the fact that, as indicated above, one of the $\mathrm{OH}$ bonds of the molecule is not available, since it points outside the water to the corresponding wall or another kind of physical limit.

\section{Concluding remarks}

Studying liquid water modeled by realistic SPC flexible and Lennard-Jones potential models inside narrow slit pores of graphene or inside carbon nanotube model devices is of great relevance from the nanotechnological point of view. In this review, we intended to compare structure, dynamical and dielectric properties and energy profiles of such systems, focusing our analysis on the reliability of our potential models within several thermodynamic conditions.

The calculations have been performed by molecular dynamics simulations of water at temperatures between 250 and $773 \mathrm{~K}$ and a wide density range. Our results of energy and free energy profiles indicate the existence of several stable phases, usually being associated with low and high densities. Within the high density phase, we define regions (interfaces, bulk) where water behaves in qualitatively different ways. For instance, at water-carbon interfaces, the hydrogen-bond network typical of unconstrained bulk water formed by four hydrogen bonds per molecule tends to weaken, losing one bond on average. In addition, in the adsorbed water layers two main orientations appear, one with both the molecular HH direction and the molecular dipole moment nearly parallel to the graphene surface and a second one with one particular oxygenhydrogen molecular bond nearly normal to the graphene plane, the so-called dangling hydrogen.

A relevant aspect concerning the dielectric properties of water at carbon-based interfaces is the dramatic rise of the permittivity, which largely fluctuates and can reach values up to a factor of five larger than in the bulk internal regions. Finally, we should mention the changes observed at the $\mathrm{OH}-$ stretching band of hydrogen, computed by Fourier transform of atomic time correlation functions. In the case of water under confinement, a splitting of that band is clearly observed (maxima at $3400 \mathrm{~cm}^{-1}$ and $3750 \mathrm{~cm}^{-1}$ ), mainly caused by the larger amount of non-hydrogen bonded hydrogens at the interfaces. Such an effect was first reported in 2001 [41] for water inside narrow carbon nanotube models and experimentally observed by Kolesnikov et al [35] two years later.

\section{Acknowledgments}

This paper is a contribution that concerns recent progresses in the field of computer simulations of water discussed at the CECAM workshop 'Modeling and Simulation of Water at Interfaces from Ambient to Supercooled Conditions' supported by ESF-Simbioma and CECAM.

MCG gratefully acknowledges financial support from the Junta de Andalucia (PAI-205). JM thanks financial support from the Direcció General de Recerca de la Generalitat de Catalunya (grant 2009SGR1003) and the Spanish Ministry of Education and Science (grant FIS2009-13641-C02-01).

\section{References}

[1] Gileadi E, Kirowa-Eisner E and Penciner J 1975 Interfacial Electrochemistry (Reading, MA: Addison-Wesley)

[2] Thiel P A and Madey T E 1987 Surf. Sci. Rep. 7 211-385

[3] Turner J E, Hendewerk M and Somorjai G A 1984 Chem. Phys. Lett. 105581

[4] Pruppacher H R and Klett J D 1978 Microphysics of Clouds and Precipitation (Dordrecht: Reidel) pp 257-68

[5] Butler G and Ison H C K 1966 Corrosion and its Prevention in Waters (New York: Reinhold)

[6] Barton K 1976 Protection Against Atmospheric Corrosion: Theories and Methods (London: Wiley)

[7] Don J A and Scholten J J F 1971 Faraday Discuss. Chem. Soc. 71145

[8] Kim J, Lu W, Qiu W, Wang L, Caffey M and Zhong D 2006 J. Phys. Chem. B $11021994-2000$

[9] Gelb L D, Gubbins K E, Radhakrishnan R and Sliwinska-Bartowiak M 1999 Rep. Prog. Phys. 621573

[10] Evans R, Marini Bettolo Marconi U and Tarazona P 1986 J. Chem. Phys. 842376

[11] Ball P C and Evans R 1989 Langmuir 5714

[12] Lee C Y, McCammon J A and Rossky P J 1984 J. Chem. Phys. 804448

[13] Zhu S-B and Robinson G W 1990 J. Chem. Phys. 941403

[14] Mamatkulov S I, Khabibullaev P K and Netz R R 2004 Langmuir 204756

[15] Choudhury N and Pettitt B M 2004 J. Am. Chem. Soc. 1273556

[16] Choudhury N and Pettitt B M 2005 J. Phys. Chem. B 1096422

[17] Rovere M, Ricci M A, Vellati D and Bruni F 1998 J. Chem. Phys. 1089859

[18] Spohr E 1990 Chem. Phys. 14187

[19] Rustad J R, Felmy A R and Bylaska E J 2003 Geochim. Cosmochim. Acta $\mathbf{6 7} 1001$

[20] Martins L R, Skaf M S and Ladanyi B M 2004 J. Phys. Chem. B 10819687

[21] Leng Y and Cummings P T 1993 Phys. Rev. Lett. 702313

[22] Gordillo M C and Martí J 2002 J. Chem. Phys. 1173425

[23] Striolo A, Chialvo A, Cummings P T and Gubbins K E 2003 Langmuir 198583 
[24] Cabrera Sanfelix P, Holloway S, Kolasinski K W and Darling G R 2003 Surf. Sci. 532-535 166

[25] Pertsin A and Grunze M 2004 J. Phys. Chem. B 1081357

[26] Gordillo M C, Nagy G and Martí J 2005 J. Chem. Phys. 123054707

[27] Hummer G, Rasaiah J C and Noworyta J P 2001 Nature 414188

[28] Gordillo M C and Martí J 2003 Phys. Rev. B 67205425

[29] Striolo A, Chialvo A, Cummings P T and Gubbins K E 2006 J. Chem. Phys. 124074710

[30] Zangi R 2004 J. Phys.: Condens. Matter 16 S5371-88

[31] Nagy G 1996 J. Electroanal. Chem. 40919

[32] Teschke O and de Souza E F 2005 Chem. Phys. Lett. 40395

[33] Sliwinska-Bartkoviak M, Jazdzewska M, Huan L L and Gubbins K E 2008 Phys. Chem. Chem. Phys. 104909

[34] Janiak C and Scharmann T G 2002 J. Am. Chem. Soc. 12414010

[35] Kolesnikov A I, Zanotti J-M, Loong C-K, Thiyagarajan P, Moravsky A P, Loufty R O and Burnham C J 2004 Phys. Rev. Lett. 93035503

[36] Ricci M A, Bruni F, Gallo P, Rovere M and Soper A K 2000 J. Phys.: Condens. Matter 12 A345

[37] Tombari E, Salvetti G, Ferrari C and Johari G P 2005 J. Chem. Phys. 122104712

[38] Scodinu A and Fourkas J T 2002 J. Phys. Chem. B 10610292

[39] Geim A K 2009 Science 3241530

[40] Liu P, Harder E and Berne B J 2004 J. Phys. Chem. B 1086595

[41] Martí J and Gordillo M C 2001 Phys. Rev. B 63165430

[42] Martí J and Gordillo M G 2001 Phys. Rev. E 64021504

[43] Martí J and Gordillo M G 2001 J. Chem. Phys. 11410492

[44] Martí J, Padró J A and Guàrdia E 1994 J. Mol. Liq. 6217

[45] Toukan K and Rahman A 1985 Phys. Rev. B 312643

[46] Martí J, Guàrdia E and Padró J A 1994 J. Chem. Phys. 10110883

[47] Martí J 1999 J. Chem. Phys. 1106876
[48] Gordillo M C and Martí J 2000 Chem. Phys. Lett. 329341

[49] Berendsen H J C, Postma J P M, van Gunsteren W F, Dinola A and Haak J R J 1984 J. Chem. Phys. 813684

[50] Iijima S 1991 Nature 35456

[51] Harris P J F 2004 Carbon Nanotubes and Related Structures (Cambridge: Cambridge University Press)

[52] Stan G and Cole M W 1998 Surf. Sci. 395280

[53] Striolo A, Chialvo A A, Gubbins K E and Cummings P T 2005 J. Chem. Phys. 122234712

[54] Hanasaki I and Nakatani A 2006 J. Chem. Phys. 124174714

[55] Takaiwa D, Hatano I, Koga K and Tanaka H 2008 Proc. Natl Acad. Sci. 10539

[56] Mann D J and Halls M D 2003 Phys. Rev. Lett. 90195503

[57] Gordillo M C and Martí J 2001 Chem. Phys. Lett. 341250

[58] Martí J and Gordillo M C 2002 Chem. Phys. Lett. 354227

[59] Martí J, Guárdia E and Gordillo M C 2002 Chem. Phys. Lett. 365536

[60] Martí J and Gordillo M C 2003 J. Phys. Chem. 11912540

[61] Gordillo M C and Martí J 2007 Phy. Rev. B 75085406

[62] Martí J, Nagy G, Gordillo M C and Guàrdia E 2006 J. Chem. Phys. 124094703

[63] Martí J, Nagy G, Guàrdia E and Gordillo M C 2006 J. Phys. Chem. B 11023987

[64] Nagy G, Gordillo M C, Guàrdia E and Martí J 2007 J. Phys. Chem. B 11112524

[65] Koga K and Tanaka H 2005 J. Chem. Phys. 122104711

[66] Koga K, Tanaka H and Cheng X C 2000 Nature 408564

[67] Brovchenko I, Geiger A and Olekinova A 2004 J. Chem. Phys. 1201958

[68] Jedlovszky P, Vincze A and Horvai G 2002 J. Chem. Phys. 1172271

[69] Pertsin A and Grunze M 2004 J. Phys. Chem. B 1081357

[70] Gordillo M C and Martí J 2008 Phys. Rev. B 78075432

[71] Martí J, Sala J, Guàrdia E and Gordillo M C 2009 Phys. Rev. E 79031606

[72] Ballenegger V and Hansen J P 2005 J. Chem. Phys. 122114711 